\title{
The neuropsychological assessment battery (NAB) is a valuable tool for evaluating neuropsychological outcome after aneurysmatic subarachnoid hemorrhage
}

\author{
Johannes Walter", Martin Grutza, Lidia Vogt, Andreas Unterberg and Klaus Zweckberger
}

\begin{abstract}
Background: Detecting and treating neuropsychological deficits after aneurysmatic subarachnoid hemorrhage (aSAH) play a key role in regaining independence; however, detecting deficits relevant to social and professional reintegration has been difficult and optimal timing of assessments remains unclear. Therefore, we evaluated the feasibility of administering the Neuropsychological Assessment Battery screening module (NAB-S) to patients with aSAH, assessed its value in predicting the ability to return to work and characterized clinical as well as neuropsychological recovery over the period of 24 months.

Methods: A total of 104 consecutive patients treated for aSAH were recruited. After acute treatment, follow up visits were conducted at 3,12 and 24 months after the hemorrhage. NAB-S, Montreal Cognitive Assessment (MoCA) and physical examination were performed at each follow up visit.

Results: The NAB-S could be administered to $64.9,75.9$ and $88.9 \%$ of the patients at 3, 12 and 24 months, respectively. Moderate impairment of two or more neuropsychological domains (e.g speech, executive function, etc.) significantly correlated with inability to return to work at 12 and 24 months as well as poor outcome assessed by the extended Glasgow Outcome Scale (GOSE) at 3, 12 and 24 months. The number of patients with favorable outcomes significantly increased from $25.5 \%$ at discharge to 56.5 and $57.1 \%$ at 3 and 12 months, respectively, and further increased to $74.1 \%$ after 24 months.

Conclusion: The NAB-S can be administered to the majority of patients with aSAH and can effectively detect clinically relevant neuropsychological deficits. Clinical recovery after aSAH continues for at least 24 months after the hemorrhage which should be considered in the design of future clinical trials.
\end{abstract}

Keywords: Subarachnoid hemorrhage, Aneurysmatic subarachnoid hemorrhage, Hemorrhagic stroke, Aneurysm, Neuropsychology, Neuropsychological assessment battery

\footnotetext{
* Correspondence: Johannes.walter@med.uni-heidelberg.de

Department of Neurosurgery, University of Heidelberg, Im Neuenheimer Feld 400, 69120 Heidelberg, Germany
}

(c) The Author(s). 2020 Open Access This article is licensed under a Creative Commons Attribution 4.0 International License, which permits use, sharing, adaptation, distribution and reproduction in any medium or format, as long as you give appropriate credit to the original author(s) and the source, provide a link to the Creative Commons licence, and indicate if changes were made. The images or other third party material in this article are included in the article's Creative Commons licence, unless indicated otherwise in a credit line to the material. If material is not included in the article's Creative Commons licence and your intended use is not permitted by statutory regulation or exceeds the permitted use, you will need to obtain permission directly from the copyright holder. To view a copy of this licence, visit http://creativecommons.org/licenses/by/4.0/. The Creative Commons Public Domain Dedication waiver (http://creativecommons.org/publicdomain/zero/1.0/) applies to the data made available in this article, unless otherwise stated in a credit line to the data. 


\section{Background}

Aneurysmatic subarachnoid hemorrhage (aSAH) is caused by rupture of an intracranial aneurysm and leads to significant long-term morbidity. As many cases of aSAH occur at a relatively young age with peak incidence at 55 years of age, many survivors are faced with limitations in their everyday life for decades [1-4]. Besides physical impairments, neuropsychological deficits have been recognized as one of the main factors affecting patient's quality of life; however, even though many outcome assessment tools like the Montreal Cognitive Assessment (MoCA) and the Mini-Mental Status Evaluation (MMSE), different memory tests like the Wechsler Memory Scale or the California Verbal Learning Test as well as tests of executive function, such as the Wisconsin Card Sorting Task, have been evaluated, it has been difficult to recognize subtle cognitive deficits by means of gross neurological measures [5-10]. Therefore, it is the goal of the current study to evaluate the feasibility of administering the Neuropsychological Assessment Battery screening module (NAB-S) to patients with aSAH. The NAB is a scale specifically developed to neuropsychologically evaluate neurologically impaired patients. It consists of a screening module which is used in the current study and five additional modules that evaluate the five domains screened with the screening module in more detail. Unlike other tests used to evaluate distinct neurocognitive domains like executive or memory function that only provide information on one single domain, the NAB-S provides information on five different domains (attention, language, memory, spatial und executive function) in a single test battery. On the other hand, it provides more detailed information than other combined test batteries that also evaluate multiple domains like the MMSE or the MoCA. Therefore, the NAB-S is a highly interesting new tool for evaluating multiple domains of neuropsychological function in a short period of time with only one single test battery.

In addition to evaluating the feasibility of applying the NAB-S to patients with aSAH, we aimed at characterizing the course of clinical and neuropsychological recovery within the first 2 years after aSAH. Many large clinical studies only evaluated outcome at 3 months after aSAH; however, significant recovery might continue for at least 24 months $[4,11-16]$. Therefore, in our study patients were followed up for at least 24 months in order to develop a clinically significant follow up plan that might influence future clinical study design.

\section{Methods}

\section{Patients}

After approval of the protocol by the local standing committee on ethical practice of the University of Heidelberg (Ethics Committee of the Medical Faculty,
University of Heidelberg, Heidelberg, Germany, Approval Number S-642/2018), a total of 104 consecutive patients treated for aneurysmatic subarachnoid hemorrhage at our academic neurosurgical clinic, which is part of a large university hospital, were prospectively recruited after providing written informed consent in accordance with the Declaration of Helsinki. If their clinical condition hampered patients from providing written consent themselves it was obtained from legal proxies. All patients were treated on the neurosurgical intensive care unit according to local standard of care consisting of rapid diagnostics including native computed tomography (CT), CT angiography and digital subtraction angiography (DSA) as well as securing the ruptured aneurysm by either clipping or coiling after interdisciplinary case discussion and, if necessary, diversion of cerebrospinal fluid (CSF) followed by neurointensive care treatment. All patients received physiotherapy while being hospitalized in our institution and were referred to postacute neurorehabilitation thereafter.

\section{Follow up}

Follow up consultations were scheduled 3, 12 and 24 months after the hemorrhage. As 20 patients died during the acute phase on the intensive care unit (ICU) and four more patients had not reached the first follow time point at the time of data analysis a maximum of $80 \mathrm{pa}-$ tients was available for neurological and neuropsychological outcome assessment. Prior to follow up patients or their legal proxies were contacted by phone to evaluate whether extensive neuropsychological examination was possible. If patients were able to follow simple commands they were scheduled for clinical and neuropsychological follow up. Otherwise a structured telephone interview consisting of the standardized GOSE telephone interview based on the suggestions published by Wilson et al. in 1998 and additional medical questions was conducted [17]. At follow up visits clinical examination including a thorough neurological assessment as well as administration of the MoCA test and the German version of the NAB-S were performed. Neurological assessments were conducted by a physician while neuropsychological assessments were conducted by a clinical neuropsychologist, who was blinded to the patients` treatment and clinical course at all follow-up visits.

\section{Montreal cognitive assessment (MoCA)}

The MoCA is a cognitive screening tool that was originally developed in 2005 to assist physicians in diagnosing mild cognitive impairment [18]. It consists of 13 tasks and evaluates multiple domains of cognitive function (visuospatial abilities, attention, concentration, language, shortterm and working memory). It can be administered within 
$15 \mathrm{~min}$ and has been used to screen patients with a wide range of conditions including aSAH [19-22]. The maximum score is 30 points and using a cutoff of 25 points is suggested to detect mild cognitive impairment; therefore, in the current study a MoCA score of 25 or less was categorized as impaired.

\section{Neuropsychological assessment battery (NAB)}

The Neuropsychological Assessment Battery is a comprehensive, modular test battery which was developed to evaluate neuropsychological function in neurologically impaired patients [23]. The initial standard random sample consisted of 1.448 individuals aged 18 to 97 . As age-, education- and gender-corrected norms were developed, it can be applied to a wide range of patients. The NAB consists of six modules: A screening module briefly evaluating each of the five domains attention, language, memory, spatial und executive function, as well as five additional modules each of which evaluates one of the domains in more detail. Different modules can be combined to create an individual neuropsychological assessment tailored to the patient's deficits and span of attention. In the current study, the German version of the NAB screening module (NAB-S) was used [24-26]. It can be applied within 45 min which is an appropriate timeframe for patients who have suffered from aSAH and rendered feasible in a clinical setting. In contrast to many tests that have previously been used to assess neuropsychological function in patients with aSAH, like the Wechsler Memory Scale or the California Verbal Learning Test, which only assess one neurocognitive domain, the NAB-S provides multidimensional information on neuropsychological function [26-28]. In addition, it provides more detailed information than other screening tests like the MoCA or the MMSE combining detailed information on all domains of neuropsychological function despite being a screening test that can be applied within a short period of time [29]. Finally, the NAB-S is well suited for repeat testing as two parallel test versions are available making it suitable for long term outcome assessments.

In the current study, the NAB-S was administered by a clinical neuropsychologist at 3,12 and 24 months after the hemorrhage. The patient's performance was compared to age-, education-, and gender-corrected norms and index values were determined for each tested domain. Finally, the results were stratified into six categories according to the index values: severe impairment, moderate impairment, mild impairment, average performance, performance above average and exceptional performance. Mild impairment was present if the patient's index value was within one standard deviation below average while test results were classified as moderately impaired if index values were between one and two standard deviations below average. If patients scored more than two standard deviations below average their performance was classified as severely impaired. Results were stratified as above average if scores were within one standard deviation above average and an exceptional performance was present if patients scored more than one standard deviation above average.

\section{Statistical analysis}

Continuous variables were compared using one way ANOVA for normally distributed variables or MannWhitney-U-Test for nonnormally distributed variables. Discrete variables were compared using $\mathrm{Chi}^{2}$ or Fisher's exact test when appropriate. Statistical significance was defined as $p \leq 0.05$.

\section{Results}

Baseline characteristics

Baseline characteristics are shown in Table 1. Mean age was $56.4+/-13.7$ years and $43.3 \%$ of the patients were female. $56.7 \%$ of the patients had low grade aSAH (WFNS grades I-III) while $43.3 \%$ of the patients suffered from poor grade aSAH (WFNS grades IV and V). In $90.7 \%$ of the cases the source of bleeding was located in the anterior circulation while $9.3 \%$ of the ruptured aneurysms were located in the posterior circulation. The imbalance in favor of anterior circulation aneurysms is due to the fact that in our facility patients with aSAH are treated on both the neurosurgical and the neurological ICU; however, patients with aneurysms more likely to be clipped which are aneurysms of the anterior circulation in most cases are preferentially treated at the neurosurgical ICU. Mean aneurysm size was $6.7+/-3.8 \mathrm{~mm}$ and significantly higher in poor grade aSAH compared to low grade aSAH $(8.0+/-4.4 \mathrm{~mm}$ vs. $5.8+/-3.1 \mathrm{~mm}$, $p=0.009$ ).

\section{Treatment and ICU stay}

In $8.4 \%$ of the patients the aneurysm was not treated due to poor clinical prognosis. In three cases two aneurysms were diagnosed and consecutively treated in the same patient. The aneurysms were treated by clipping in $42.9 \%$ and by coiling in $57.1 \%$ of the cases. Mean length of stay on the ICU or intermediate care unit (IMC) was $21.2+/-10.5$ days. Patients with low grade aSAH were treated on the ICU or IMC for a significantly shorter period of time than patients suffering from poor grade aSAH $(18.9+/-10.3$ days vs. $24.9+/-9.7$ days, $p<0.001)$. During ICU treatment $56.7 \%$ of the patients developed delayed cerebral ischemia (DCI) which was defined as new focal neurological deficit persisting for more than $1 \mathrm{~h}$, drop of oxygen pressure in the cerebral tissue (pTiO2) below $15 \mathrm{mmHg}$ determined by intraparenchymal pTiO2probe, acceleration of mean velocity in the middle cerebral 
Table 1 Baseline characteristics of the enrolled patients

\begin{tabular}{|c|c|c|c|c|}
\hline & Total & WFNS I-III & WFNS IV + V & $p$-value \\
\hline Number & $100 \%(104 / 104)$ & $56.7 \%(59 / 104)$ & $43.3 \%(45 / 104)$ & \\
\hline Age (years) & $56.4+/-13.7$ & $57.8+/-15.2$ & $54.5+/-11.3$ & 0.22 \\
\hline Female sex & $43.3 \%(45 / 104)$ & $47.5 \%(28 / 59)$ & $37.8 \%(17 / 45)$ & 0.43 \\
\hline Male sex & $56.7 \%(59 / 104)$ & $52.5 \%(31 / 59)$ & $61.2 \%(28 / 45)$ & 0.43 \\
\hline Anterior circulation aneurysma & $90.7 \%(97 / 107)$ & $93.5 \%(58 / 62)$ & $86.7 \%(39 / 45)$ & 0.43 \\
\hline Posterior circulation aneurysma & $9.3 \%(10 / 107)$ & $6.5 \%(4 / 62)$ & $13.3 \%(6 / 45)$ & 0.43 \\
\hline Mean aneurysm size (mm) & $6.7+/-3.8$ & $5.8+/-3.1$ & $8.0+/-4.4$ & 0.009 \\
\hline
\end{tabular}

an three cases of low grade aSAH more than one aneurysm was detected by initial angiography

artery (MCA) above $160 \mathrm{~cm} / \mathrm{s}$ determined by transcranial doppler sonography, cerebral perfusion deficit determined by CT angiography or cerebral vasospasm diagnosed by DSA. All cases of DCI were treated with elevation of mean arterial pressure (MAP); furthermore, in $76.6 \%$ of the patients with DCI intraarterial Nimodipin was administered and percutaneous balloon angioplasty for treatment of macro-vasospasm was performed in $4.3 \%$ of the cases. Decompressive hemicraniectomy for refractory elevated intracranial pressure was performed in $19.4 \%$ of the cases. Total in hospital mortality was $19.2 \%$ dropping to $10.6 \%$ when only accounting for cases with initial treatment of the ruptured aneurysm. In hospital mortality in low grade aSAH was significantly lower than in poor grade aSAH ( $8.5 \%$ vs. $33.3 \%, p=0.003)$. Median Glasgow Coma Score (GCS) at discharge was 13.0. Median Extended Glasgow Outcome Scale (GOSE) score at discharge was 3.0 with $25.5 \%$ of the patients reaching a favorable outcome of
GOSE score five or better and $29.5 \%$ of the patients scoring 3 or better on the modified Rankin Scale (mRS). In low grade $\mathrm{SAH}$, median GCS and GOSE scores at discharge were 15 and 4.5 , respectively, with $47.1 \%$ of the patients reaching a GOSE score of five or more and 50.0\% scoring 3 or better on the mRS. In poor grade $\mathrm{SAH}$, median GCS and mean GOSE scores at discharge were 9.0 and 2.0, respectively. No patient suffering from poor grade SAH reached a GOSE score of five or more and only $4.7 \%$ scored 3 or better on the mRS at discharge, indicating a significantly worse outcome at discharge compared to low grade aSAH $(p<0.001$ for both comparisons, Table 2).

\section{Clinical outcome at follow up}

The number of patients with a GOSE score of five or better indicating independence at home but inability to return to work significantly increased to 56.5 and $57.1 \%$

Table 2 Treatment characteristics and in-hospital outcome. WFNS: World Federation of Neurological Surgery, DCl: Delayed cerebral ischemia, ICU: Intensive care unit, GCS: Glasgow Coma Score, GOSE: Glasgow Outcome Score Extended, mRS: Modified Rankin Scale

\begin{tabular}{|c|c|c|c|c|}
\hline & Total & WFNS I-III & WFNS IV + V & $p$-value \\
\hline Clipping $^{a}$ & $42.9 \%(42 / 98)$ & $39.3 \%(24 / 61)$ & $48.6 \%(18 / 37)$ & 0.49 \\
\hline Coiling $^{a}$ & $57.1 \%(56 / 98)$ & $60.6 \%(37 / 61)$ & $51.4 \%(19 / 37)$ & 0.49 \\
\hline No Treatment ${ }^{\mathrm{b}}$ & $8.4 \%(9 / 107)$ & $1.6 \%(1 / 62)$ & $17.8 \%(8 / 45)$ & 0.009 \\
\hline $\mathrm{DCl}$ & $56.7 \%(47 / 83)$ & $49.1 \%(26 / 53)$ & $70.0 \%(21 / 30)$ & 0.11 \\
\hline Intraarterial Nimodipine for DCl & $76.6 \%(36 / 47)$ & $73.1 \%(19 / 26)$ & $81.0 \%(17 / 21)$ & 0.73 \\
\hline Balloon Angioplasty for DCl & $4.3 \%(2 / 47)$ & $7.7 \%(2 / 26)$ & $0 \%(0 / 21)$ & 0.50 \\
\hline Decompressive Hemicraniectomy & $19.4 \%(19 / 98)$ & $11.5 \%(7 / 61)$ & $32.4 \%(12 / 37)$ & 0.02 \\
\hline Mean ICU stay (days) & $21.2+/-10.5$ & $18.9+/-10.3$ & $24.9+/-9.7$ & $<0.001$ \\
\hline In-hospital mortality & $19.2 \%(20 / 104)$ & $8.5 \%(5 / 59)$ & $33.3 \%(15 / 45)$ & 0.003 \\
\hline GCS at discharge (median) & $13.0(3-15)$ & $15.0(3-15)$ & $9.0(3-15)$ & \\
\hline GOSE at discharge (median) & $3.0(1-8)$ & $4.5(1-8)$ & $2.0(1-4)$ & \\
\hline mRS at discharge (median) & $5.0(0-6)$ & $3.0(0-6)$ & $5.0(3-6)$ & \\
\hline GOSE $\geq 5$ & $25.5 \%(24 / 94)$ & $47.1 \%(24 / 51)$ & $0 \%(0 / 43)$ & $<0.001$ \\
\hline $\mathrm{mRS} \leq 3$ & $29.5 \%(28 / 95)$ & $50.0 \%(26 / 52)$ & $4.7 \%(2 / 43)$ & $<0.001$ \\
\hline
\end{tabular}

In three cases of low grade aSAH two aneurysms were treated within the initial ICU stay because the source of bleeding could not be determined with certainty in two cases an in one case a second aneurysm was located in close proximity to the ruptured one

$\mathrm{b} / \mathrm{n}$ one case of low grade aSAH treatment was intentionally delayed because of presence of severe vasospasm on initial angiogram (the patient was admitted 8 days after the hemorrhage). The patient died due to fulminant pulmonary embolism 10 days later before the aneurysm had been secured 
at 3 and 12 months, respectively $(p<0.001$ and $p=$ 0.002 , respectively) indicating significant neurological improvement within the first 12 months after the hemorrhage. Between 12 and 24 months, the number of patients with a GOSE score of five or more further increased to $74.1 \%$ indicating a trend towards further functional recovery in the second year after the bleeding. Patients with low grade aSAH were significantly more likely to reach a GOSE of 5 or better after 3 months compared to patients with poor grade aSAH (77.8\% vs. $26.3 \%, p=0.002)$. At 12 months this difference could also be detected (73.7\% vs. $37.5 \%, p=0.07$ ).

There was a significant increase in number of patients with an mRS score of three or less indicating the ability to walk without assistance but requiring some help with daily activities at 3 and 12 months compared to discharge (69.6 and $68.6 \%$ vs. $29.5 \%, p<0.001$ for both comparisons) indicating significant neurological improvement within the first 12 months after bleeding. Between 12 and 24 months, the number of patients with a $\mathrm{mRS}$ score of three or more further increased to $77.8 \%$. At 3 months the number of patients with a mRS of 3 or better was significantly higher in low grade aSAH compared to poor grade aSAH (85.2\% vs. $47.4 \%, p=0.016)$. This difference was reduced at 12 and 24 months $(78.9 \%$ vs. 56.3 and $87.5 \%$ vs. $63.6 \%$, respectively, Table 3 ).

\section{Neuropsychological outcome}

NAB screening was not possible due to clinical condition in $35.1 \%$ of the patients at 3 months. The number of patients in which NAB screening was impossible due to clinical condition decreased throughout the first 24 months $(24.1$ and $11.1 \%$ at 12 and 24 months, respectively).

If medium or more severe impairments were present in two or more domains of the NAB-S significantly more patients had a GOSE score of 4 or less compared to patients without these impairments at 3, 12 and 24 months (66.7\% vs. $0 \%, p=0.003 ; 66.7 \%$ vs. $7.7 \%, p=0.017$ and $75.0 \%$ vs. $0 \%, \mathrm{p}=0.003$, respectively).

Patients with intraparenchymal or intraventricular blood clots (Fisher ${ }^{\circ} \mathrm{IV}$ ) were more likely to have two or more moderately impaired domains of the NAB at 12 months compared to patients with subarachnoid blood clots only (Fisher ${ }^{\circ} \mathrm{I}-\mathrm{III} ; 44.4 \%$ vs. $0 \%$ ), however, this difference leveled off by 24 months (30.8\% vs. $20.0 \%$ ).

Patients with DCI were more likely to have moderate impairment of at least two domains of the NAB-S at 24 months $(50.0 \%$ vs. $12.5 \%)$, however, this difference did not reach statistical significance due to small number of patients. There was no difference between the two groups at 12 months (36.4\% vs. $30.0 \%)$.

At 3 and 12 months, 31.4 and $33.3 \%$ of the patients scored 26 or more points in the MoCA and therefore had an unimpaired MoCA score. The number of patients with unimpaired MoCA scores increased to $41.7 \%$ at 24 months.

\section{Return to work}

Data on occupational status is summarized in Table 3. No patient was able to return to work at 3 months. After 12 months, $23.5 \%$ of the patients had returned to work indicating a significant increase $(p<0.001)$. After 24 months the number of patients who had returned to work further increased to $37.5 \%$. Low grade aSAH was associated with significantly higher numbers of patients who had returned to work at 12 months compared to poor grade aSAH (36.8\% vs. $6.7 \%, p=0.05)$. This trend persisted at 24 months ( $53.8 \%$ vs $18.2 \%)$.

There was no severe impairment of any domain of the NAB-S in any of the patients who had been able to return to work at any timepoint after the hemorrhage. If no severe impairment was present in any domain of the NAB-S significantly more patients were able to return to work at 12 and 24 months compared to patients with severe impairment of any NAB-S domain ( $0 \%$ vs. $50.0 \%$, $p=0.047$ and $0 \%$ vs. $57.1 \%, p=0.045$, respectively).

No patient with at least medium impairment of two or more NAB-S domains was able to return back to work at either 3, 12 or 24 months. Significantly less patients were able to return to work at 12 and 24 months if medium impairment of two or more NAB-S domains were present ( $0 \%$ vs. $50.0 \%, p=0.047$ and $0 \%$ vs. $57.1 \%$, $p=0.045$, respectively).

At 12 months, one patient with an impaired MoCA score of 25 points and at 24 months two patients with impaired MoCA tests were able to return to work. Patients were more likely to be able to return to work at 12 months if they had scored 26 points or more compared to patients who scored less than 26 points $(57.1 \%$ vs. $14.3 \%$ ); however, this difference was not statistically significant due to small patient number. The number of unimpaired MoCA scores in the group of patients who had been able to return to work was not different from the group of patients who had not been able to return to work at 24 months ( $40.0 \%$ vs. $22.2 \%)$. Table 4 summarizes the correlation between neuropsychological outcome and the ability to return to work.

\section{Discussion}

\section{Clinical outcome}

It has been noted that neuropsychological deficits have a significant impact on patients` recovery from aSAH [30]. In our study, moderate impairment of neuropsychological function was also significantly correlated to poor outcome assessed by GOSE at 3, 12 and 24 months after the hemorrhage. On one hand, this underscores the importance of neuropsychological assessment as part of 


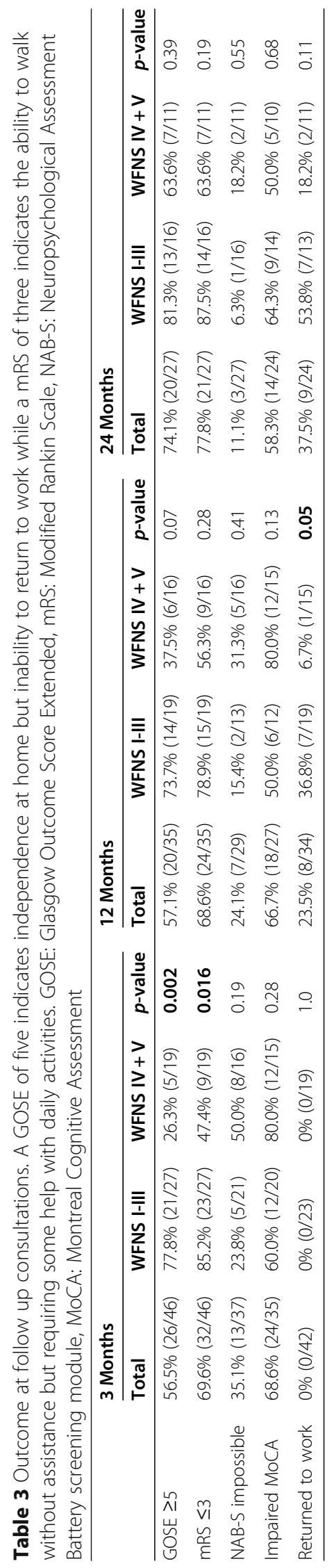


Table 4 Correlation between neuropsychological outcome and the ability to return to work. The NAB-S assesses the five domains attention, language, memory, spatial und executive function. MoCA was considered impaired when scoring less than 26 points. NAB-S: Neuropsychological Assessment Battery screening module, MoCA: Montreal Cognitive Assessment

\begin{tabular}{|c|c|c|c|c|c|c|c|c|c|c|}
\hline & & \multicolumn{3}{|c|}{$\begin{array}{l}\text { Medium impairment of NAB-S } \\
\text { domains }\end{array}$} & \multicolumn{3}{|c|}{$\begin{array}{l}\text { Severe impairment of NAB-S } \\
\text { domains }\end{array}$} & \multicolumn{3}{|l|}{ MoCA } \\
\hline & & $<2$ & $\geq 2$ & $p$-value & $<1$ & $\geq 1$ & $p$-value & impaired & unimpaired & $p$-value \\
\hline \multirow[t]{2}{*}{ Return to work } & 12 months & $50.0 \%(7 / 14)$ & $0 \%(0 / 7)$ & 0.047 & $50.0 \%(7 / 14)$ & $0 \%(0 / 7)$ & 0.047 & $14.3 \%(1 / 7)$ & $57.1 \%(4 / 7)$ & 0.266 \\
\hline & 24 months & $57.1 \%(8 / 14)$ & $0 \%(0 / 5)$ & 0.045 & $57.1 \%(8 / 14)$ & $0 \%(0 / 5)$ & 0.045 & $22.2 \%(2 / 9)$ & $40.0 \%(2 / 5)$ & 0.580 \\
\hline
\end{tabular}

follow up schedules for patients with aSAH to detect deficits that might be amenable to therapeutic intervention. On the other hand, we could show that the NAB-S can effectively detect neuropsychological deficits relevant for recovery in a cohort of patients with aSAH, and therefore is a promising tool for implementation in $\mathrm{SAH}$ follow up strategies.

The presence of intraparenchymal or intraventricular blood clots on initial CT imaging was associated with moderate impairment of at least two domains of the NAB-S at 12 months in our study. This is well in line with the results of previous studies indicating a relationship between the initial Fisher score and neuropsychological deficits and highlights the importance of blood location and clot size for neuropsychological outcome [31-35]. This might be due to the relation of Fisher grade and development of delayed cerebral ischemia; however, in our study, the development of DCI did not significantly correlate with more severe neuropsychological impairment indicating an important role of parenchymal lesions due to initial intraparenchymal hemorrhage and chronic hydrocephalus in the development of neuropsychological deficits within the first 12 months after the bleeding. However, as more patients with DCI developed neuropsychological deficits 24 months after the hemorrhage, DCI seems to play an important role in the development of neuropsychological deficits in the chronic phase after aSAH.

\section{Neuropsychological outcome}

The NAB was developed to evaluate neurologically impaired patients aged 18 to 97 [23]. In clinical practice, the NAB screening module has only been administered to small inhomogeneous cohorts of patients with mixed neurological pathologies such as traumatic brain injury or brain tumors [36]. To our knowledge, in the current study it was systematically administered to patients with aSAH for the first time. In our cohort the NAB-S could be administered to 75.9 and $88.9 \%$ of the patients at 12 and 24 months, respectively. Therefore, administration of the NAB-S at 12 and 24 months after aSAH is well feasible, however, as only $50.0 \%$ of the patients with high grade aSAH could be tested at 3 months, the battery is probably more useful for low grade aSAH patients at this timepoint.

\section{Return to work}

Returning to work is a very important goal of rehabilitation and social reintegration for patients after suffering from aSAH; however, in our study population only a small number of patients who returned for follow up returned to work to their previous capacity $(0,23.5$ and $37.5 \%$ at 3,12 and 24 months, respectively) which is well in line with previous reports [30, 37, 38]. Therefore, predicting the ability to return to work is a very important requirement for a neuropsychological assessment tool to be of clinical use. In our study, no patient who returned to work had severe impairments of any or moderate impairments of two or more domains of the NAB-S. If a severe deficit was present at 12 or 24 months the patient was significantly less likely to return to work. Furthermore, the patient was also less likely to return to work if two or more domains were moderately impaired at 12 or 24 months. Therefore, the NAB-S performed very well in detecting neuropsychological deficits that were relevant for predicting a patient's ability to return to work at previous capacity. This is of even more interest as Wallmark et al. reported that return to work could be predicted using the MoCA [37]. However, Wallmark et al. did not report data on MoCA scores and occupational status at 24 months and were able to correctly predict occupational status in only $68 \%$ of the cases. In our study, non-impaired MoCA scores of 26 points or more did not significantly correlate with a higher likelihood to return to work at any timepoint and there were patients who had returned to work to their previous capacity despite having scored 25 or less points on the MoCA at 12 as well as 24 months after the bleeding. Therefore, we feel that the MoCA test is not useful to detect relevant neuropsychiological deficits prohibiting patients from returning to work and that using the NAB-S generates important additional information on the patient's neuropsychological status and ability to return to work compared to the MoCA.

\section{Neurological recovery following aSAH}

In addition to neuropsychological assessments, the current study prospectively evaluated clinical outcome 
up to 24 months after aSAH. It could be shown that significant recovery assessed by GOSE which has been the most important outcome parameter in large clinical trials continues throughout the first 2 years after the bleeding. However, many large randomized clinical trials which failed to show any beneficial effects of various therapeutic strategies for aSAH evaluated outcome at 3 months only $[4,11-16]$. Therefore, effects on long term outcome might have been missed due to too short follow up. Future studies should account for the fact that clinical recovery continues for at least 24 months after aSAH and include longer follow up schedules of at least 12 or if possible 24 months.

\section{Limitations}

In the current study, due to the acute nature of aSAH no information on premorbid neurocognitive function can be presented, as extensively evaluating patients 'neurocognitive function before an acute event is naturally not possible. However, this might affect evaluation of neuropsychological outcome at the follow-up visits as patients might have been impaired even before the acute event of aSAH. Furthermore, numbers of patients especially at later stages of follow-up are limited as we present our first single center experiences with the NAB-S in patients with aSAH. Due to this, exact analysis of temporal patterns of intraindividual functional and neuropsychological recovery, which would of course be of great interest, is significantly hampered. Finally, at 3 months after the hemorrhage, the NAB-S could not be administered to a third of the patients due to clinical condition; therefore, the use of the NAB-S in all aSAH patients at this timepoint cannot be generally recommended and might be more appropriate in low grade aSAH patients only.

\section{Conclusion}

In the current study, the NAB screening module was used to neuropsychologically assess patients with aSAH for the first time. It could be administered to the majority of patients at 12 and 24 months after SAH; therefore, clinical use is feasible. At 3 months, the battery is probably more useful for low grade aSAH patients as at this timepoint only $50 \%$ of the high grade SAH patients could be tested. Neuropsychological deficits detected by the NAB-S were significantly correlated to patients' ability to return to work as well as clinical outcome assessed by GOSE; therefore, it provided valuable additional information on patients` functional status. Finally, it could be shown that recovery after aSAH continues for at least 24 months after the hemorrhage; therefore, in order to detect long term therapeutic effects future clinical studies investigating aSAH should assess clinical outcome for a longer period of time as it has been the case in the past.

\begin{abstract}
Abbreviations
aSAH: Aneurysmatic subarachnoid hemorrhage; CSF: Cerebrospinal fluid; CT: Computed tomography; DCl: Delayed cerebral ischemia; DSA: Digital subtraction angiography; GCS: Glasgow Coma Scale; GOSE: Extended

Glasgow Outcome Scale; ICU: Intensive care unit; IMC: Intermediate care unit; mRS: Modified Rankin Scale; MoCA: Montreal Cognitive Assessment; MMSE: Mini-Mental Status Evaluation; NAB: Neuropsychological Assessment Battery; NAB-S: Neuropsychological Assessment Battery Screening Module; pTiO2: Brain tissue partial oxygen pressure; SAH: Subarachnoid hemorrhage; WFNS: World Federation of Neurological Surgeons
\end{abstract}

\section{Acknowledgements \\ We acknowledge the help of Maria Holz in study coordination. \\ Authors' contributions \\ Conceptualization: JW and KZ; Methodology: JW, KZ and LV; Software: JW; Validation: KZ, AU and LV; Formal Analysis: KZ and MG; Investigation: JW, MG and LV; Resources: KZ and AU; Data Curation: JW and MG; Writing-Original Draft Preparation: JW; Writing — Review and Editing: KZ, LV and AU; Visualization: JW; Supervision: KZ and AU; Project Administration: $\mathrm{MH}$; Funding Acquisition: KZ and AU. All authors have read and approved the manuscript.}

\section{Funding}

No funding was received for this research. Open Access funding enabled and organized by Projekt DEAL.

\section{Availability of data and materials}

The datasets used and/or analysed during the current study are available from the corresponding author on reasonable request.

\section{Ethics approval and consent to participate}

All procedures performed in studies involving human participants were in accordance with the ethical standards of the institutional and/or national research committee and with the 1964 Helsinki declaration and its later amendments or comparable ethical standards. After approval of the protocol by the local standing committee on ethical practice of the University of Heidelberg (Approval Number S-642/2018), written informed consent was obtained from all individual participants or their legal guardians included in the study. Ethics Committee of the Medical Faculty, University of Heidelberg, Heidelberg, Germany, Approval Number S-642/2018

\section{Consent for publication}

Not applicable.

\section{Competing interests}

All authors certify that they have no affiliations with or involvement in any organization or entity with any financial interest (such as honoraria; educational grants; participation in speakers' bureaus; membership, employment, consultancies, stock ownership, or other equity interest; and expert testimony or patent-licensing arrangements), or non-financial interest (such as personal or professional relationships, affiliations, knowledge or beliefs) in the subject matter or materials discussed in this manuscript.

Received: 16 June 2020 Accepted: 17 November 2020

Published online: 26 November 2020

\footnotetext{
References

1. Macdonald RL, Schweizer TA. Spontaneous subarachnoid haemorrhage. Lancet. 2017;389(10069):655-66.

2. Schweizer TA, Macdonald RL. Brain hemorrhage: assessing outcome after subarachnoid hemorrhage. Nat Rev Neurol. 2010;6(8):427-8.

3. Al-Khindi T, Macdonald RL, Schweizer TA. Cognitive and functional outcome after aneurysmal subarachnoid hemorrhage. Stroke. 2010;41(8):e519-36.

4. Eagles ME, Tso MK, Macdonald RL. Cognitive Impairment, Functional Outcome, and Delayed Cerebral Ischemia After Aneurysmal Subarachnoid Hemorrhage. World Neurosurg. 2019.

5. Koso M, Dizdarevic K, Sose-Selimotic J. Everyday memory in microsurgically treated patients after subarachnoid hemorrhage. J Clin Med Res. 2015;7(4): 225-31.
} 
6. Koso M, Dizdarevic K. Attention and executive functions in microsurgically treated patients after subarachnoid hemorrhage. Asian J Neurosurg. 2015; 10(4):260-5

7. Dey S, Kumar JK, Shukla D, Bhat D. Neurological, neuropsychological, and functional outcome after good grade aneurysmal subarachnoid hemorrhage. Neurol India. 2018;66(6):1713-7.

8. Otawara Y, Ogasawara K, Kubo Y, Kashimura H, Ogawa A, Yamadate K. Comparison of postoperative cognitive function in patients undergoing surgery for ruptured and unruptured intracranial aneurysm. Surg Neurol. 2009;72(6):592-5 discussion 5.

9. Joswig H, Korte W, Fruh S, Epprecht L, Hildebrandt G, Fournier JY, et al Neurodegenerative cerebrospinal fluid biomarkers tau and amyloid beta predict functional, quality of life, and neuropsychological outcomes after aneurysmal subarachnoid hemorrhage. Neurosurg Rev. 2018;41(2): 605-14.

10. Scott RB, Farmer E, Smiton A, Tovey C, Clarke M, Carpenter K. Methodology of neuropsychological research in multicentre randomized clinical trials: a model derived from the international subarachnoid aneurysm trial. Clin Trials. 2004;1(1):31-9.

11. Macdonald RL, Kassell NF, Mayer S, Ruefenacht D, Schmiedek P, Weidauer S, et al. Clazosentan to overcome neurological ischemia and infarction occurring after subarachnoid hemorrhage (CONSCIOUS-1): randomized, double-blind, placebo-controlled phase 2 dose-finding trial. Stroke. 2008; 39(11):3015-21.

12. Macdonald RL, Higashida RT, Keller E, Mayer SA, Molyneux A, Raabe A, et al. Randomized trial of clazosentan in patients with aneurysmal subarachnoid hemorrhage undergoing endovascular coiling. Stroke. 2012;43(6):1463-9.

13. Macdonald RL, Higashida RT, Keller E, Mayer SA, Molyneux A, Raabe A, et al. Clazosentan, an endothelin receptor antagonist, in patients with aneurysmal subarachnoid haemorrhage undergoing surgical clipping: a randomised, double-blind, placebo-controlled phase 3 trial (CONSCIOUS-2). Lancet Neurol. 2011;10(7):618-25.

14. Chou SH, Smith EE, Badjatia N, Nogueira RG, Sims JR 2nd, Ogilvy CS, et al. A randomized, double-blind, placebo-controlled pilot study of simvastatin in aneurysmal subarachnoid hemorrhage. Stroke. 2008;39(10):2891-3.

15. Dorhout Mees SM, Algra A, Wong GK, Poon WS, Bradford CM, Saver JL, et al. Early magnesium treatment after aneurysmal subarachnoid hemorrhage: individual patient data meta-analysis. Stroke. 2015:46(11):3190-3.

16. Wong GK, Chan DY, Siu DY, Zee BC, Poon WS, Chan MT, et al. High-dose simvastatin for aneurysmal subarachnoid hemorrhage: multicenter randomized controlled double-blinded clinical trial. Stroke. 2015;46(2):382-8.

17. Wilson JT, Pettigrew LE, Teasdale GM. Structured interviews for the Glasgow outcome scale and the extended Glasgow outcome scale: guidelines for their use. J Neurotrauma. 1998;15(8):573-85.

18. Nasreddine ZS, Phillips NA, Bedirian V, Charbonneau S, Whitehead V, Collin I, et al. The Montreal cognitive assessment, MoCA: a brief screening tool for mild cognitive impairment. J Am Geriatr Soc. 2005;53(4):695-9.

19. Stienen MN, Fung C, Bijlenga P, Zumofen DW, Maduri R, Robert T, et al. Measuring the impact of delayed cerebral ischemia on neuropsychological outcome after aneurysmal subarachnoid hemorrhage-protocol of a Swiss Nationwide observational study (MoCA-DCl study). Neurosurgery. 2019;84(5): 1124-32.

20. Zaki Ghali MG, Srinivasan VM, Wagner K, Rao C, Chen SR, Johnson JN, et al. Cognitive Sequelae of Unruptured and ruptured intracranial aneurysms and their treatment: modalities for neuropsychological assessment. World Neurosurg. 2018;120:537-49.

21. De Roeck EE, De Deyn PP, Dierckx E, Engelborghs S. Brief cognitive screening instruments for early detection of Alzheimer's disease: a systematic review. Alzheimers Res Ther. 2019;11(1):21.

22. Skorvanek M, Feketeova E, Kurtis MM, Rusz J, Sonka K. Accuracy of rating scales and clinical measures for screening of rapid eye movement sleep behavior disorder and for predicting conversion to Parkinson's disease and other Synucleinopathies. Front Neurol. 2018;9:376.

23. White T, Stern R. Neuropsychological assessment battery. Lutz, Florida, USA: Psychological Assessment Resources; 2003.

24. Buczylowska D, Bornschlegl M, Daseking M, Jäncke L, Petermann F. Zur deutschen adaptation der neuropsychological assessment battery (NAB). Z Neuropsychol. 2013;24:217-27.

25. Petermann F, Jäncke L. Neuropsychological Assessment Battery (NAB) Aussagekraft und Anwendungen der deutschsprachigen Adaptation. Z Neuropsychol. 2016;27:129-31.
26. Bornschlegl M, Speer P, Danneil W, Vogt T, Petermann F. Interne Validität der Neuropsychological Assessment Battery (NAB): Zusammenhang der Testwerte im Screening mit den vertiefenden Modulen bei Patienten mit Schlaganfall und Gesunden. Z Neuropsychol. 2016;27:133-46.

27. Powell J, Kitchen N, Heslin J, Greenwood R. Psychosocial outcomes at 18 months after good neurological recovery from aneurysmal subarachnoid haemorrhage. J Neurol Neurosurg Psychiatry. 2004;75(8):1119-24.

28. Elwood RW. The California verbal learning test: psychometric characteristics and clinical application. Neuropsychol Rev. 1995;5(3):173-201.

29. McLennan SN, Mathias JL, Brennan LC, Stewart S. Validity of the Montreal cognitive assessment (MoCA) as a screening test for mild cognitive impairment $(\mathrm{MCl})$ in a cardiovascular population. J Geriatr Psychiatry Neurol. 2011:24(1):33-8.

30. Buunk AM, Groen RJM, Wijbenga RA, Ziengs AL, Metzemaekers JDM, van Dijk JMC, et al. Mental versus physical fatigue after subarachnoid hemorrhage: differential associations with outcome. Eur J Neurol. 2018; 25(11):1313-e113.

31. Orbo M, Waterloo K, Egge A, Isaksen J, Ingebrigtsen T, Romner B. Predictors for cognitive impairment one year after surgery for aneurysmal subarachnoid hemorrhage. J Neurol. 2008;255(11):1770-6.

32. Hutter BO, Kreitschmann-Andermahr I, Gilsbach JM. Cognitive deficits in the acute stage after subarachnoid hemorrhage. Neurosurgery. 1998;43(5):1054-65.

33. Larsson C, Forssell A, Ronnberg J, Lindberg M, Nilsson LG, Fodstad H. Subarachnoid blood on CT and memory dysfunctions in aneurysmal subarachnoid hemorrhage. Acta Neurol Scand. 1994;90(5):331-6.

34. Stienen MN, Weisshaupt R, Fandino J, Hildebrandt G, Studerus-Germann A Schatlo B. Characteristics of patients without neuropsychological deficits following aneurysmal subarachnoid haemorrhage. Acta Neurochir Suppl. 2015;120:125-9.

35. de Souza ML, Vieira AC, Andrade G, Quinino S. de Fatima Leal Griz M, Azevedo-Filho HR. Fisher grading scale associated with language disorders in patients with anterior circulation aneurysmal subarachnoid hemorrhage. World Neurosurg. 2015;84(2):308-13.

36. Iverson GL, Williamson DJ, Ropacki M, Reilly KJ. Frequency of abnormal scores on the neuropsychological assessment battery screening module (S-NAB) in a mixed neurological sample. Appl Neuropsychol. 2007;14(3):178-82.

37. Wallmark S, Ronne-Engstrom E, Lundstrom E. Predicting return to work after subarachnoid hemorrhage using the Montreal cognitive assessment (MoCA). Acta Neurochir. 2016;158(2):233-9.

38. Buunk AM, Groen RJ, Veenstra WS, Spikman JM. Leisure and social participation in patients 4-10 years after aneurysmal subarachnoid haemorrhage. Brain Inj. 2015;29(13-14):1589-96.

\section{Publisher's Note}

Springer Nature remains neutral with regard to jurisdictional claims in published maps and institutional affiliations.
Ready to submit your research? Choose BMC and benefit from:

- fast, convenient online submission

- thorough peer review by experienced researchers in your field

- rapid publication on acceptance

- support for research data, including large and complex data types

- gold Open Access which fosters wider collaboration and increased citations

- maximum visibility for your research: over $100 \mathrm{M}$ website views per year

At $\mathrm{BMC}$, research is always in progress.

Learn more biomedcentral.com/submissions 